\title{
Zoneamento do potencial de uso de sistemas de resfriamento evaporativo no sudeste brasileiro
}

\author{
Valcimar F. Carvalho ${ }^{1}$, Tadayuki Yanagi Junior ${ }^{1}$, Leandro Ferreira ${ }^{1}$, Flávio A. Damasceno ${ }^{1}$ \& Mariano P. Silva ${ }^{2}$
}

\section{RESUMO}

O zoneamento do potencial de uso de sistemas de resfriamento evaporativo (SRE) acoplado a um sistema de ventilação em modo túnel (SVT) foi realizado no presente trabalho, com vistas à criação de frangos de corte na região Sudeste do Brasil. Quatro configurações do sistema foram testadas: a) SVT; b) SVT associado à SRE do tipo material poroso umedecido com $40 \%$ de eficiência $\left(S_{\text {PAD }}\right)$; c) SVT associado à SRE do tipo material poroso umedecido com $70 \%$ de eficiência $\left(\mathrm{SVT}_{\text {PAD70}}\right)$ e d) $S V \mathrm{~T}_{\text {PAD70 }}$ associado a sistema de nebulização $\left(S V T_{\text {PAD70+NEB }}\right)$. O ambiente térmico foi caracterizado por meio do índice de temperatura e umidade, enquanto o desempenho das aves foi determinado pelo consumo de ração diário, ganho de peso diário e conversão alimentar. Dados meteorológicos de 1980 a 2001, disponibilizados pelo Projeto Reanálise-2 do NCEP/NCAR, foram utilizados nesta pesquisa. Verificou-se o efeito benéfico do uso dos SRE, com $\mathrm{SVT}_{\text {PAD70+NEB }}$ proporcionando as melhores condições de produção, em especial nos meses de setembro e outubro para quase toda a região Sudeste, com destaque para as mesorregiões Norte e Noroeste de Minas Gerais.

Palavras-chave: nebulização, índice de conforto, frango de corte

\section{Mapping of potential use of evaporative cooling systems in Southeastern Brazil}

\begin{abstract}
In present research the potential use of evaporative cooling systems (ECS) associated to tunnel ventilation system (TVS) for broiler reared in Southeastern Brazil was studied. Four configuration systems were tested: (a) TVS, (b) TVS associated to a pad cooling system with $40 \%$ efficiency $\left(T_{V S} S_{\text {PAD40 }}\right.$ ), (c) TVS associated to a pad cooling system with $70 \%$ efficiency

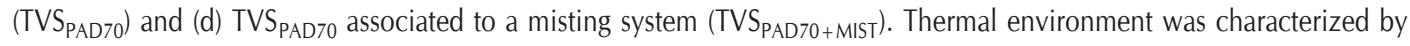
temperature-humidity index, and the chicken performance was estimated by daily feed intake, daily weight gain and feed conversion. Climatic data from 1980 to 2001, released through Reanalysis-2 Project from NCEP/NCAR were used in this research. The benefitial effect of the use of ECS with TVS PAD70+MIST $_{\text {for providing better production condition was }}$ verified, especially during September and October for almost all the southeastern region, mainly in northern and northwestern part of the Minas Gerais State.
\end{abstract}

Key words: fogging, thermal index, poultry 


\section{INTRODUÇÃO}

A exemplo de Brasil, em climas tropicais e subtropicais, os elevados valores de temperatura de bulbo seco do ar (Tbs) se encontram entre os principais fatores que interferem negativamente na produção de frangos de corte (Tinôco, 1996). Na fase adulta a ave se adapta melhor a ambientes frios, visto que seu sistema termorregulador é mais adequado para reter calor que para dissipá-lo. Quando exposta a estresse térmico por elevadas temperaturas, a ave apresenta diminuição no consumo de ração e, em conseqüência, redução no ganho de peso e pior conversão alimentar (Bueno, 2004). Tendo em vista este aspecto, diversas formas de arrefecimento do ar têm sido propostas para as instalações avícolas, desde o uso otimizado dos recursos da própria natureza até métodos artificiais, como a ventilação e o resfriamento evaporativo (Baêta \& Souza, 1997).

A ventilação lateral, ou a ventilação em modo túnel, com pressão positiva ou negativa, promove a dissipação de calor sensível no interior da instalação; entretanto, este efeito benéfico se torna nulo quando a Tbs se equipara à temperatura corporal da ave, havendo a necessidade do pré-resfriamento do ar a ser circulado no ambiente. O uso de sistemas de resfriamento evaporativo (SRE) possibilita a redução da Tbs em até $11^{\circ} \mathrm{C}$ em algumas regiões. No Brasil, a média desta redução é de $6^{\circ} \mathrm{C}$ (Tinôco, 1996).

Diversos estudos envolvendo os efeitos da ventilação, do resfriamento evaporativo e da densidade de alojamento, têm sido realizados na busca do entendimento e de soluções de problemas de campo (Corria \& Nääs, 2001; Sartor et al., 2001; Stowell et al., 2001; Tinôco et al., 2004); no entanto, para se viabilizar economicamente estudos mais complexos, poder-se-á utilizar modelos matemáticos e computacionais, como forma de se obter redução de tempo e de custos no desenvolvimento e adaptação de projetos, permitindo estudar aspectos estruturais e o desempenho dos animais.

Com os avanços dos sistemas de informações geográficas (SIG) e a maior disponibilidade de dados meteorológicos, tornou-se possível fazer o zoneamento bioclimático de determinada região (Gates et al., 1995; Severo et al., 2003; Oliveira, 2004; Silva et al., 2005; Turco et al., 2006) ou, ainda, gerar mapas de alerta associados à previsão do tempo.

Objetivou-se, assim,com o presente trabalho, fazer o zoneamento do potencial de uso de sistemas de resfriamento evaporativo (SREs) acoplado a um sistema de ventilação em modo túnel, com pressão negativa, para a criação de frangos de corte na região Sudeste do País.

\section{MATERIAL E MÉTODOS}

\section{Dados climatológicos}

Para as estimativas do índice de temperatura e umidade - ITU e do desempenho dos frangos de corte, obtidos por meio do consumo de ração diário (CRD, $\left.\mathrm{g} \mathrm{d}^{-1}\right)$, ganho de peso diário (GPD, $\mathrm{g} \mathrm{d}^{-1}$ ) e conversão alimentar (CA, $\mathrm{g} \mathrm{g}^{-1}$ ), no interior de galpões climatizados se utilizaram, para a região Sudeste do Brasil, dados de Tbs, umidade relativa (UR) e pressão atmosférica $\left(\mathrm{P}_{\mathrm{atm}}\right)$ do projeto denominado Reanalysis-2, do National Centers for Environmental Prediction (NCEP) e do National Center for Atmospheric Research (NCAR), para o período compreendido entre janeiro de 1980 e dezembro de 2001, em intervalos de uma hora.

Neste estudo utilizou-se uma malha constituída de noventa pontos (90 coordenadas), sendo dez pontos igualmente espaçados para a Longitude, variando de $37^{\circ} 30^{\prime} 00^{\prime \prime}$ a $54^{\circ} 22^{\prime} 30^{\prime \prime} \mathrm{O}$ e nove pontos igualmente espaçados para a latitude, variando de $12^{\circ} 22^{\prime} 6^{\prime \prime}$ a $27^{\circ} 37^{\prime} 7^{\prime}$ ' S, extrapolando a região Sudeste e propiciando maior precisão na geração das isolinhas.

Os valores horários de Tbs e UR ao longo do dia foram estimados com base na metodologia proposta por Zolnier (1996) e usada por Oliveira (2004) e Silva et al. (2005).

\section{Simulação computacional}

As estimativas do ambiente térmico e do desempenho produtivo dos frangos de corte ao longo do comprimento do galpão foram feitas para os 22 anos de dados climatológicos (1980-2001), em horários compreendidos entre 10 e 18 h, para as 90 coordenadas. A escolha desse intervalo (8 h) coincide com o período mais quente do dia, em que o funcionamento dos SRE é praticamente contínuo.

Nas simulações se utilizaram o modelo matemático e o programa computacional SisTunel 1.0, proposto por Carvalho (2006). O SisTunel simula o ambiente térmico e o desempenho de frangos de corte, ao longo do comprimento do galpão, calculando-se os valores do índice de temperatura e umidade (ITU), por meio da Eq. 1, proposta por Thom (1959), em cada volume de controle tal como, também, os valores do CRD, GPD e CA, pelas equações 2, 3 e 4, respectivamente, proposto por Medeiros (2001).

$$
\mathrm{ITU}_{\mathrm{i}}=\mathrm{t}_{\mathrm{bsi}}+0,36 \cdot \mathrm{t}_{\mathrm{poi}}+41,5
$$

em que:

$\mathrm{ITU}_{\mathrm{i}}$ - Índice de temperatura e umidade no i-ésimo volume de controle

tbs i - Temperatura de bulbo seco no i-ésimo volume de controle, ${ }^{\circ} \mathrm{C}$

tpo i - Temperatura de ponto de orvalho no i-ésimo volume de controle, ${ }^{\circ} \mathrm{C}$

$$
\begin{aligned}
\mathrm{CRD}_{\mathrm{i}}= & 28,963697+11,306258 \cdot \mathrm{t}_{\mathrm{bsi}}+0,03095 \cdot \mathrm{UR}_{\mathrm{i}} \\
& -6,89328 \cdot \mathrm{V}_{\mathrm{i}}-0,25476 \cdot \mathrm{t}_{\mathrm{bsi}}{ }^{2}+0,002513 \cdot \mathrm{UR}_{\mathrm{i}}{ }^{2} \\
& +1,3084 \cdot \mathrm{V}_{\mathrm{i}}{ }^{2}-0,01389 \cdot \mathrm{t}_{\mathrm{bsi}} \cdot \mathrm{UR}_{\mathrm{i}}+0,24676 \cdot \mathrm{t}_{\mathrm{bsi}} \cdot \mathrm{V}_{\mathrm{i}} \\
& -0,02579 \cdot \mathrm{UR}_{\mathrm{i}} \cdot \mathrm{V}_{\mathrm{i}} \\
\mathrm{GPD}_{\mathrm{i}}= & -92,717789+14,292936 \cdot \mathrm{t}_{\mathrm{bsi}}+0,253649 \cdot \mathrm{UR}_{\mathrm{i}} \\
& -9,515579 \cdot \mathrm{V}_{\mathrm{i}}-0,290314 \cdot \mathrm{t}_{\mathrm{bsi}}{ }^{2}+0,001538 \cdot \mathrm{UR}_{\mathrm{i}}{ }^{2} \\
& +0,974936 \cdot \mathrm{V}_{\mathrm{i}}{ }^{2}-0,016171 \cdot \mathrm{t}_{\mathrm{bsi}} \cdot \mathrm{UR}_{\mathrm{i}} \\
& +0,294444 \cdot \mathrm{t}_{\mathrm{bsi}} \cdot \mathrm{V}_{\mathrm{i}}-0,007407 \cdot \mathrm{UR}_{\mathrm{i}} \cdot \mathrm{V}_{\mathrm{i}} \\
\mathrm{CA}_{\mathrm{i}}= & 6,364195-0,379572 \cdot \mathrm{t}_{\mathrm{bsi}}-0,00151 \cdot \mathrm{UR}_{\mathrm{i}} \\
+ & 0,268889 \cdot \mathrm{V}_{\mathrm{i}}+0,00748 \cdot \mathrm{t}_{\mathrm{bsi}}{ }^{2}-0,00004651 \cdot \mathrm{UR}_{\mathrm{i}}{ }^{2} \\
& -0,049766 \cdot \mathrm{V}_{\mathrm{i}}{ }^{2}+0,00025 \cdot \mathrm{t}_{\mathrm{bsi}} \cdot \mathrm{UR}_{\mathrm{i}}-0,00370 \cdot \mathrm{t}_{\mathrm{bsi}} \cdot \mathrm{Vi}^{\mathrm{i}} \\
& -0,00026 \cdot \mathrm{UR}_{\mathrm{i}} \cdot \mathrm{V}_{\mathrm{i}}
\end{aligned}
$$


em que:

$\mathrm{CRD}_{\mathrm{i}}$ - Consumo de ração diário de cada ave no i-ésimo VC, $\mathrm{g} \mathrm{d}^{-1}$

$\mathrm{GPD}_{\mathrm{i}}-$ Ganho de peso diário de cada ave no i-ésimo VC, $\mathrm{g} \mathrm{d}^{-1}$

$\mathrm{CA}_{\mathrm{i}}$ - Conversão alimentar de cada ave no i-ésimo $\mathrm{VC}, \mathrm{g} \mathrm{g}^{-1}$

$\mathrm{UR}_{\mathrm{i}}$ - Umidade relativa no i-ésimo VC, \%

$\mathrm{V}_{\mathrm{i}}$ - Velocidade do ar à altura de $0,30 \mathrm{~m}$ no i-ésimo $\mathrm{VC}, \mathrm{m} \mathrm{s}^{-1}$

Quatro diferentes configurações de controle do ambiente de criação (tratamentos) de frangos de corte foram simuladas: a) apenas sistema de ventilação do tipo túnel de vento com pressão negativa - SVT (controle); b) SVT associado a sistema de resfriamento evaporativo do tipo material poroso umedecido com $40 \%$ de eficiência de resfriamento $\left(\mathrm{SVT}_{\mathrm{PAD} 40}\right)$ : c) SVT associado a sistema de resfriamento evaporativo do tipo material poroso umedecido com $70 \%$ de eficiência de resfriamento $\left(\mathrm{SVT}_{\mathrm{PAD} 70}\right)$ e d) $\mathrm{SVT}_{\mathrm{PAD70}}$ associado a sistema de nebulização $\left(\mathrm{SVT}_{\mathrm{PAD} 70+\mathrm{NEB}}\right)$.

Além dos dados de Tbs, UR e $\mathrm{P}_{\mathrm{atm}}$, obtidos do banco de dados climatológicos, utilizaram-se também, como dados de entrada, outras variáveis, sendo as principais descritas a seguir: (a) galpão com dimensões de 125 x 12 x 2,5 m, dividido em 125 volumes de controle com sistema de iluminação acionado durante $11 \mathrm{~h} \mathrm{~d}^{-1}$; (b) frangos de corte com massa corporal média igual a 2,4 kg, alojados na densidade de 14 aves $\mathrm{m}^{-2}$; (c) coeficiente global de transferência de calor da cobertura igual a $0,233 \mathrm{~W} \mathrm{~m}^{-2} \mathrm{~K}^{-1}$; (d) vazões de ar que atravessa a placa porosa umedecida e se infiltra ao longo do galpão, principalmente através das cortinas, iguais a 70 e $3,5 \mathrm{~m}^{3} \mathrm{~s}^{-1}$, respectivamente, e (e) vazão de água nebulizada no galpão igual a $420 \mathrm{~L} \mathrm{~h}^{-1}$ (no caso do $\mathrm{SVT}_{\text {PAD70+NEB }}$ ).

Adotou-se, nas simulações, o seguinte manejo do SVT e SRE: aciona-se o SVT para valores de Tbs iguais ou superiores a $25^{\circ} \mathrm{C}$, considerado limite superior da zona de conforto térmico para aves adultas (Curtis, 1983; Zulovich \& Deshazer, 1990). Em condições nas quais o SVT não é capaz de manter a Tbs abaixo de $29^{\circ} \mathrm{C}$ liga-se, então, o SRE $\left(\mathrm{SVT}_{\mathrm{PAD} 40}, \mathrm{SVT}_{\mathrm{PAD} 70}, \mathrm{SVT}_{\mathrm{PAD70+NEB}}\right.$, dependendo da simulação executada), que permanece acionado enquanto a UR no interior do galpão apresenta valores inferiores a $80 \%$, considerado limite máximo admissível de conforto às aves (Baião, 1995) e Tbs maior ou igual a $29^{\circ} \mathrm{C}$. Para valores de UR maiores que $80 \%$ ou Tbs menor que $29^{\circ} \mathrm{C}$ desliga-se o SRE, permanecendo apenas o SVT acionado.

\section{Zoneamento bioclimático}

Para a geração dos mapas mensais de isolinhas de -ITU médio, CRD médio, GPD médio e CA média para SVT, reduções de ITU médio e CA média e aumentos de GPD médio e CRD médio, proporcionados pelo $\mathrm{SVT}_{\mathrm{PAD} 40}, \mathrm{SVT}_{\mathrm{PAD70}}$ e $\mathrm{SVT}_{\mathrm{PAD70+NEB}}$ em relação ao SVT, para a região Sudeste do Brasil, utilizaram-se aplicativo Spring (Câmara et al., 1996) e o banco de dados b_atlas_access.exe do Instituto Nacional de Pesquisas Espaciais (INPE, 2006). A interpolação dos dados se deu pelo método da média simples, para uma grade retangular de 5 x $5 \mathrm{~km}$, estimando-se o valor da cota para cada ponto a partir da média simples dos oito pontos mais próximos.

Os dados dos ITU mensais foram espacializados e distribuídos em quatro classes de acordo com os intervalos propostos por Thom (1959), em que ITU menor que 74 configura conforto térmico, entre 74 e 79 representa situação de alerta, entre 79 e 84 indica situação de perigo e acima de 84 configura situação de emergência. Apesar de não ser o melhor índice para ser aplicado em estudos de ambiência avícola, o ITU proposto por Thom (1959) tem sido amplamente usado devido à disponibilidade dos dados de Tbs e UR em diversos bancos de dados, sendo recentemente usado por diversos autores (Medeiros, 2001; Silva et al., 2004; Silva et al., 2005; Oliveira et al., 2006) A finalização dos mapas gerados foi pelo módulo Scarta do aplicativo Spring.

Para a identificação das áreas classificadas utilizou-se a subdivisão em mesorregiões (Figura 1) apresentada pelo IBGE (2006).

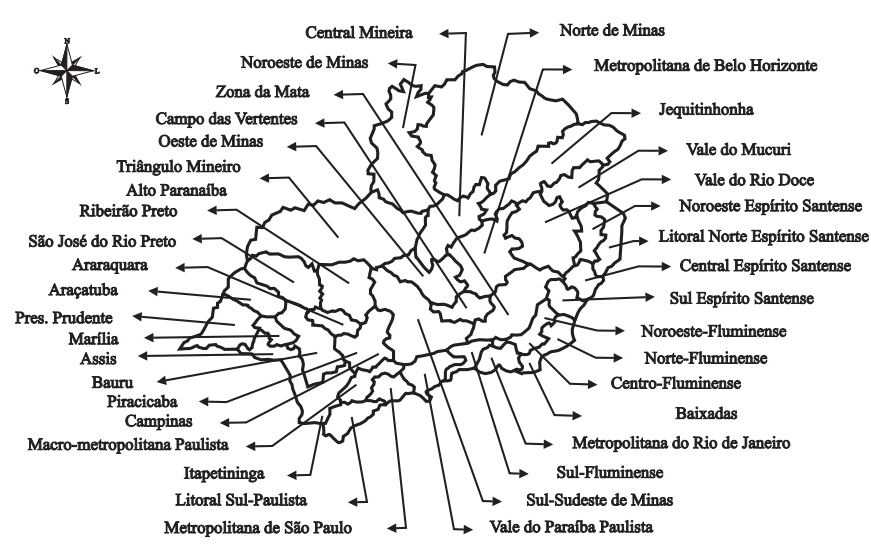

Figura 1. Distribuição das mesorregiões dos estados da região sudeste do Brasil, adaptadas do IBGE (2006)

\section{RESULTADOS E DISCUSSÃO}

\section{Ambiente térmico}

Por meio da análise do ambiente térmico no interior do galpão, no horário entre 10 e 18 h, verificou-se que o tratamento SVT apresentou valores médios de ITU dentro da faixa classificada alerta aos produtores (Thom, 1959), ou seja, entre 74 e 79, para todos os meses do ano (Tabela 1).

O uso de SRE associado ao SVT, em suas várias configurações $\left(\mathrm{SVT}_{\mathrm{PAD} 40}, \mathrm{SVT}_{\mathrm{PAD} 70}\right.$ e $\left.\mathrm{SVT}_{\mathrm{PAD} 70+\mathrm{NEB}}\right)$, fez com que o ITU médio reduzisse de 0,2 a 1,9. Neste trabalho, o período mais crítico para a criação de frangos de corte ocorreu entre os meses de outubro a março, enquanto Oliveira (2004) observou o mesmo comportamento entre os meses de dezembro a março;essas diferenças se devem, provavelmente, ao fato de que Oliveira (2004) considerou apenas valores de Tbs e UR para o ambiente externo e utilizou dados de ITU ao longo do dia (24 h) no cálculo das médias obtendo, assim, valores inferiores aos simulados neste trabalho. Silva et al. (2005) verificaram que o mês mais crítico é janeiro, porém neste trabalho os meses de janeiro e fevereiro apresentaram 
Tabela 1. Valores médios mensais de ITU, CRD $\left(\mathrm{g} \mathrm{d}^{-1}\right)$, GPD $\left(\mathrm{g} \mathrm{d}^{-1}\right)$ e CA $\left(\mathrm{g} \mathrm{g}^{-1}\right)$, para o tratamento SVT e reduções de ITU e CA e aumentos de CRD e GPD, para os tratamentos $S V T_{\text {PAD40, }} S_{\text {PAD70 }}$ e $S V T_{\text {PAD70+NEB }}$ em relação ao SVT, calculados para o período de 10 e $18 \mathrm{~h}$

\begin{tabular}{|c|c|c|c|c|c|c|c|c|c|c|c|c|c|}
\hline & \multicolumn{12}{|c|}{ Mês } \\
\hline & & Jan & Fev & Mar & Abr & Mai & Jun & Jul & Ago & Set & Out & Nov & Dez \\
\hline \multirow{4}{*}{ SVT } & ITU & $78,3 \pm 0,7$ & $78,4 \pm 0,6$ & $78,1 \pm 0,6$ & $77,5 \pm 0,6$ & $76,8 \pm 0,6$ & $76,2 \pm 0,5$ & $75, \pm 0,6$ & $76,2 \pm 0,8$ & $77,2 \pm 1,1$ & $77,9 \pm 1,2$ & $77,9 \pm 1,0$ & $78,0 \pm 0,9$ \\
\hline & CRD & $133,8 \pm 2,9$ & $133,8 \pm 2,2$ & $134,6 \pm 2,0$ & $135,9 \pm 1,6$ & $137,0 \pm 1,5$ & $137,9 \pm 1,3$ & $138,0 \pm 1,7$ & $136,4 \pm 3,4$ & $133,6 \pm 5,2$ & $132,3 \pm 5,4$ & $133,3 \pm 4,3$ & $133,9 \pm 3,7$ \\
\hline & GPD & $72,9 \pm 2,6$ & $72,8 \pm 2,0$ & $73,5 \pm 1,8$ & $74,4 \pm 1,4$ & $75,1 \pm 1,3$ & $75,4 \pm 1,2$ & $75,3 \pm 1,7$ & $73,9 \pm 3,2$ & $71,6 \pm 4,7$ & $70,8 \pm 4,8$ & $71,9 \pm 3,8$ & $72,7 \pm 3,3$ \\
\hline & $\mathrm{CA}$ & $1,88 \pm 0,07$ & $1,88 \pm 0,05$ & $1,86 \pm 0,05$ & $1,84 \pm 0,04$ & $1,84 \pm 0,03$ & $1,84 \pm 0,04$ & $1,84 \pm 0,05$ & $1,88 \pm 0,08$ & $1,93 \pm 0,12$ & $1,94 \pm 0,12$ & $1,91 \pm 0,09$ & $1,88 \pm 0,08$ \\
\hline \multirow{4}{*}{ SVTPAD40 } & ITU & $0,5 \pm 0,5$ & $0,4 \pm 0,5$ & $0,4 \pm 0,4$ & $0,3 \pm 0,4$ & $0,4 \pm 0,4$ & $0,5 \pm 0,4$ & $0,6 \pm 0,5$ & $1,0 \pm 0,7$ & $1,2 \pm 0,8$ & $1,1 \pm 0,8$ & $0,8 \pm 0,6$ & $0,6 \pm 0,6$ \\
\hline & CRD & $1,9 \pm 2,1$ & $1,7 \pm 2,0$ & $1,5 \pm 1,7$ & $1,3 \pm 1,3$ & $1,41,2$ & $1,5 \pm 1,2$ & $1,9 \pm 1,7$ & $3,3 \pm 2,8$ & $4,7 \pm 3,6$ & $4,5 \pm 3,3$ & $3,4 \pm 2,6$ & $2,5 \pm 2,3$ \\
\hline & GPD & $1,7 \pm 1,8$ & $1,5 \pm 1,7$ & $1,3 \pm 1,5$ & $1,1 \pm 1,1$ & $1,1 \pm 1,0$ & $1,2 \pm 1,4$ & $1,5 \pm 1,4$ & $2,7 \pm 2,4$ & $4,0 \pm 3,2$ & $4,0 \pm 3,0$ & $3,0 \pm 2,3$ & $2,2 \pm 2,1$ \\
\hline & $\mathrm{CA}$ & $0,04 \pm 0,05$ & $0,04 \pm 0,04$ & $0,03 \pm 0,04$ & $0,03 \pm 0,03$ & $0,03 \pm 0,02$ & $0,03 \pm 0,02$ & $0,03 \pm 0,03$ & $0,06 \pm 0,06$ & $0,1 \pm 0,08$ & $0,1 \pm 0,07$ & $0,07 \pm 0,06$ & $0,05 \pm 0,05$ \\
\hline \multirow{4}{*}{ SVTPAD70 } & ITU & $0,5 \pm 0,6$ & $0,4 \pm 0,6$ & $0,3 \pm 0,5$ & $0,2 \pm 0,4$ & $0,3 \pm 0,4$ & $0,4 \pm 0,5$ & $0,9 \pm 0,8$ & $1,6 \pm 1,3$ & $1,8 \pm 1,4$ & $1,5 \pm 1,1$ & $1,0 \pm 0,8$ & $0,6 \pm 0,7$ \\
\hline & CRD & $1,8 \pm 2,4$ & $1,5 \pm 2,3$ & $1,2 \pm 1,8$ & $0,8 \pm 1,2$ & $0,9 \pm 1,1$ & $1,2 \pm 1,3$ & $2,3 \pm 2,3$ & $4,4 \pm 4,0$ & $6,1 \pm 5,0$ & $5,6 \pm 4,4$ & $3,8 \pm 3,2$ & $2,5 \pm 2,9$ \\
\hline & GPD & $1,5 \pm 2,1$ & $1,3 \pm 1,9$ & $1,0 \pm 1,5$ & $0,7 \pm 1,0$ & $0,7 \pm 0,9$ & $0,9 \pm 1,0$ & $1,7 \pm 1,7$ & $3,4 \pm 3,2$ & $4,9 \pm 4,2$ & $4,7 \pm 3,8$ & $3,2 \pm 2,8$ & $2,2 \pm 2,5$ \\
\hline & $\mathrm{CA}$ & $0,04 \pm 0,05$ & $0,03 \pm 0,05$ & $0,02 \pm 0,04$ & $0,02 \pm 0,02$ & $0,02 \pm 0,02$ & $0,02 \pm 0,02$ & $0,04 \pm, 04$ & $0,08 \pm 0,08$ & $0,12 \pm 0,1$ & $0,11 \pm 0,09$ & $0,07 \pm 0,07$ & $0,05 \pm 0,06$ \\
\hline \multirow{4}{*}{ SVTPAD70+NEB } & ITU & $0,4 \pm 0,7$ & $0,4 \pm 0,6$ & $0,3 \pm 0,5$ & $0,2 \pm 0,4$ & $0,3 \pm 0,4$ & $0,5 \pm 0,5$ & $0,9 \pm 0,9$ & $1,6 \pm 1,3$ & $1,9 \pm 1,4$ & $1,5 \pm 1,2$ & $1,0 \pm 0,9$ & $0,6 \pm 0,8$ \\
\hline & CRD & $1,7 \pm 2,4$ & $1,4 \pm 2,3$ & $1,2 \pm 1,9$ & $0,8 \pm 1,2$ & $0,9 \pm 1,2$ & $1,2 \pm 1,4$ & $2,3 \pm 2,3$ & $4,5 \pm 4,1$ & $6,1 \pm 5,0$ & $5,5 \pm 4,4$ & $3,7 \pm 3,2$ & $2,4 \pm 2,9$ \\
\hline & GPD & $1,4 \pm 2,1$ & $1,2 \pm 2,0$ & $1,0 \pm 1,6$ & $0,6 \pm 1,0$ & $0,7 \pm 0,9$ & $0,9 \pm 1,0$ & $1,7 \pm 1,8$ & $3,4 \pm 3,3$ & $5,0 \pm 4,2$ & $4,7 \pm 3,7$ & $3,1 \pm 2,7$ & $2,1 \pm 2,4$ \\
\hline & $\mathrm{CA}$ & $0,03 \pm 0,05$ & $0,03 \pm 0,05$ & $0,02 \pm 0,04$ & $0,02 \pm 0,02$ & $0,02 \pm 0,02$ & $0,02 \pm 0,02$ & $0,04 \pm 0,04$ & $0,08 \pm 0,08$ & $0,12 \pm 0,1$ & $0,11 \pm 0,09$ & $0,08 \pm 0,07$ & $0,05 \pm 0,06$ \\
\hline
\end{tabular}

os maiores valores de ITU médio, 78,3 \pm 0,7 e 78,4 $\pm 0,6$, respectivamente.

Nos meses quentes e secos, como setembro e outubro, o uso de SRE associado ao SVT, mostrou-se mais eficientes. Setembro foi o mês que apresentou as maiores reduções de ITU médio, 1,2 \pm 0,8, 1,8 \pm 1,4 e 1,9 \pm 1,4, para $\mathrm{SVT}_{\mathrm{PAD} 40}$, $\mathrm{SVT}_{\mathrm{PAD70}}$ e $\mathrm{SVT}_{\mathrm{PAD70+NEB}}$, respectivamente; esses resultados ocorreram devido à característica climática da região Sudeste que, no geral, apresenta verões quentes e úmidos, reduzindo o déficit de vapor de pressão e, conseqüentemente, limitando o potencial de evaporação de água (Yanagi Júnior et al., 2002).

Os valores médios de ITU mostrados na Tabela 1, fornecem uma visão geral do comportamento sazonal do ITU para toda a região Sudeste, porém o comportamento espacial do ITU médio é mais bem caracterizado por meio de mapas de isolinhas de ITU e isolinhas de redução de ITU, como ilustrado na Figura 2, para o mês de setembro. O maior aproveitamento dos SREs foi obtido sempre que a localização do galpão se distanciou do litoral, partindo em direção ao continente, no sentido noroeste.

Partes das mesorregiões Norte e Noroeste de Minas apresentaram os maiores potenciais de redução de ITU (de 3 a 4), seguidas de partes das mesorregiões do Jequitinhonha, Central Mineira, Oeste e Sul-Sudoeste de Minas, Triângulo Mineiro/Alto Paranaíba, Campinas, Ribeirão Preto, São José do Rio Preto, Piracicaba, Araraquara, Bauru, Araçatuba, Assis, Marília e Presidente Prudente, que mostraram reduções um pouco menores (2 a 3). Algumas mesorregiões do Sul e Noroeste Espírito Santense, Vale do Mucuri, Vale do Rio Doce, Zona da Mata, Campo das Vertentes, Sul-Sudoeste de Minas, Macro-metropolitana Paulista, Litoral Sul-Paulista, Itapetininga, Vale do Paraíba Paulista e Sul-Fluminense, apontaram reduções de ITU entre 1 e 2; para as demais mesorregiões concentradas na maior parte dos estados do Rio de Janeiro e Espírito Santo e a mesorregião Metropolitana de São Paulo, as reduções de ITU foram inferiores a 1, cu- jos resultados concordam com as normais climatológicas publicadas pelo INMET (2006), para Tbs e UR no mês de setembro, entre 1931 a 1990 (Figura 3). Na Figura 3 se observa que a parte mais quente e seca do Sudeste se localiza na região Norte de Minas, tornando-a mais propícia ao uso de SRE, uma vez que a eficiência desses sistemas é diretamente proporcional ao déficit de pressão de vapor local. Ademais e se partindo da premissa de que, para uma mesma temperatura, a eficiência dos SRE é maior a medida em que a UR se torna menor, conclui-se, pela Figura 3B, que a redução do ITU pelo uso de SRE tende a ser maior sempre que se desloca do litoral, partindo em direção ao continente, no sentido noroeste.

Os resultados aqui mostrados, diferem daqueles encontrados por Silva et al. (2005), que consideram a região costeira do Rio de Janeiro e do Espírito Santo e parte fronteiriça do Sudeste com a Bahia, como sendo as regiões com as maiores reduções de ITU, ao contrário da região central do Sudeste que, segundo os autores, é a região de menor benefício ao se fazer uso de ser, enquanto o modelo de Silva et al. (2005) simula um único valor de ITU para determinado galpão, nesta pesquisa o modelo calcula o ITU do volume de controle a posteriori, em função das variáveis ambientais obtidas no volume de controle anterior. Na verdade, as mudanças de fase do ar em cada volume de controle, são consideradas reduzindo os erros de simulação; apartir desses valores, se obtem então, o valor médio do ITU para o galpão.

Para os meses de setembro e outubro, os tratamentos $\mathrm{SVT}_{\mathrm{PAD70}}$ e $\mathrm{SVT}_{\mathrm{PAD70+NEB}}$ foram classificados os melhores em relação a $\mathrm{SVT}_{\mathrm{PAD} 40}$; no entanto, para o mês de abril o oposto foi observado para uma pequena parte da mesorregião norte de Minas, ou seja, $\mathrm{SVT}_{\mathrm{PAD} 40}$ sendo melhor que $\mathrm{SVT}_{\mathrm{PAD70}}$ e $\mathrm{SVT}_{\mathrm{PAD70+NEB}}$, cuja explicação reside no fato de que, para o período estudado, os tratamentos $\mathrm{SVT}_{\mathrm{PAD} 70}$ ou $\mathrm{SVT}_{\mathrm{PAD} 70+\mathrm{NEB}}$ foram desligados mais vezes que o $\mathrm{SVT}_{\mathrm{PAD} 40}$, devido ao manejo adotado nesses sistemas, que determina desligá-los, caso a UR no interior do galpão ultrapasse 80\%, 
ITU

A.

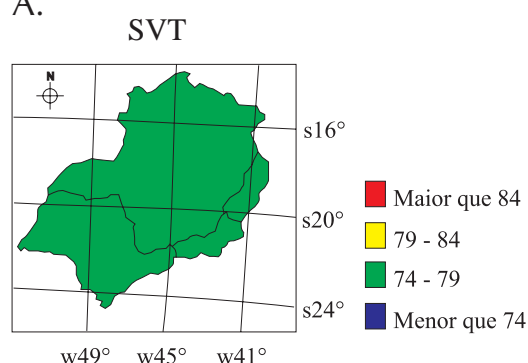

CRD

E.

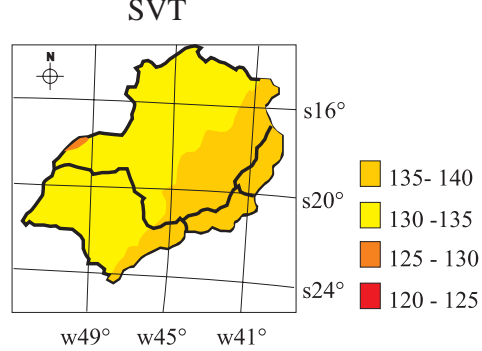

GPD

I.

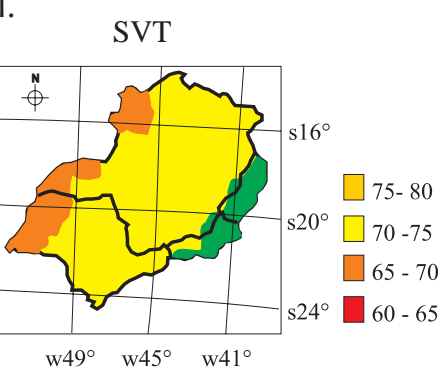

CA

M

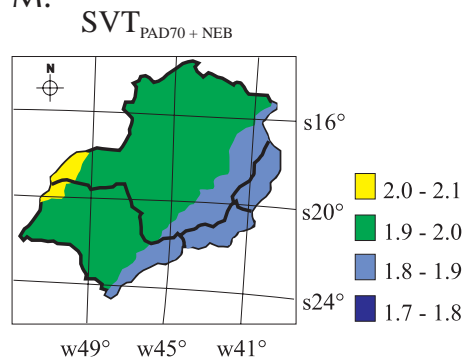

B.

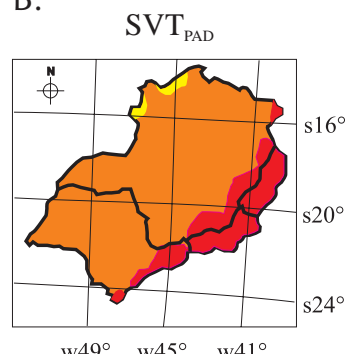

C.

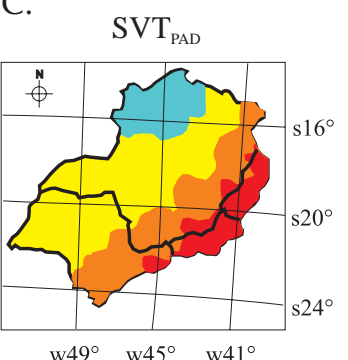

Aumento do CRD

F.

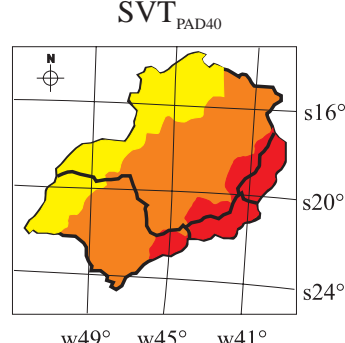

G.

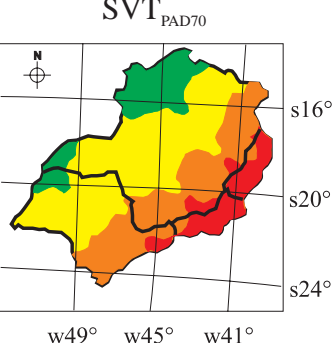

Aumento do GPD

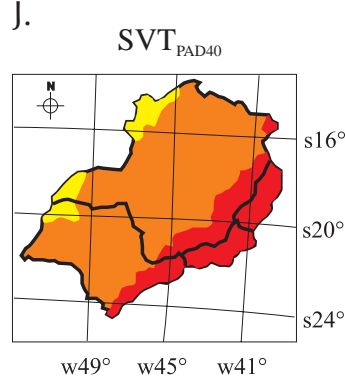

K.

N.

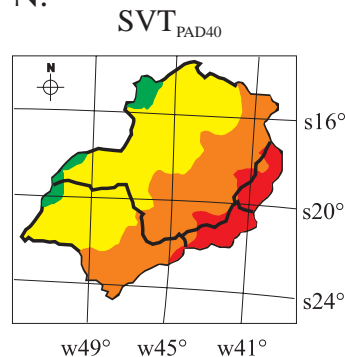

D.

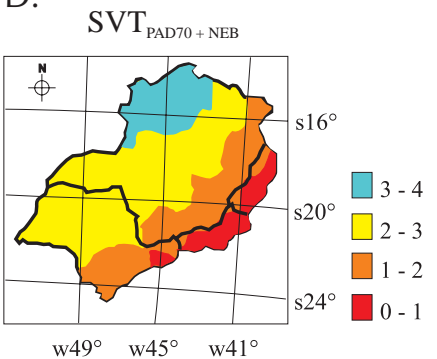

$\mathrm{H}$.

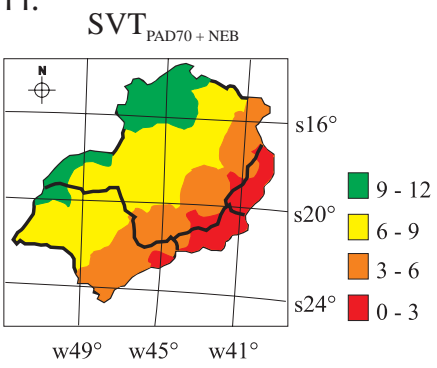

Figura 2. Distribuição espacial dos valores médios de ITU (A), CRD (E), GPD (I) e CA (M) no interior de galpões para criação de frangos de corte com ventilação em modo túnel (SVT); redução de ITU (B, C, D) e CA (N, O, P) e aumento de CRD (F, G, H) e GPD (J, K, L) para SVT associado a sistema de resfriamento evaporativo do tipo material poroso umedecido com $40 \%\left(S_{V} T_{\text {PAD 40 }}\right)$ e $70 \%$ de eficiências $\left(S_{V} T_{P A D 70}\right)$ e $S V T_{\text {PAD70 }}$ funcionando simultaneamente a sistema de nebulização $\left(\mathrm{SVT}_{\mathrm{PAD} 70+\mathrm{NEB}}\right)$, para o mês de setembro, na região Sudeste do Brasil

uma vez que, nessas condições, são maiores as dificuldades das aves em perder calor latente (Curtis, 1983; Sarmento et al., 2005; Medeiros et al., 2005; Jácome et al., 2007); além disso, sempre que a UR aumenta, para uma mesma temperatura, reduz-se o déficit de pressão de vapor mas também a capacidade do ar em absorver umidade.

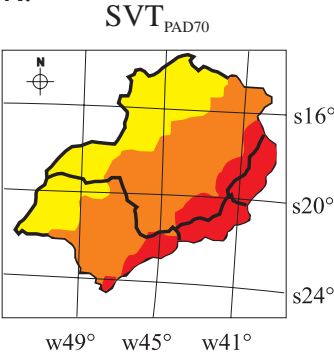

Redução da CA

O.

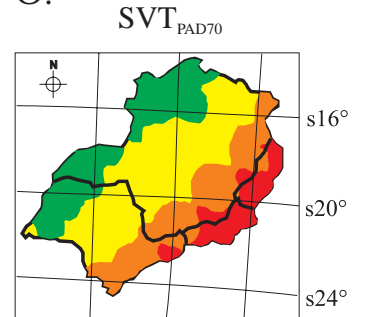

P.

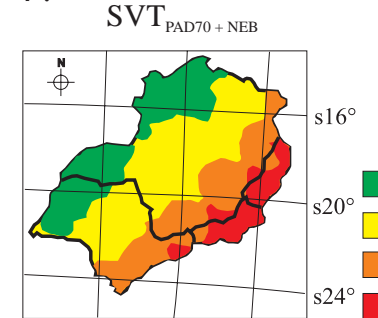

$0.15-0.20$

$0.10-0.15$

$0.05-0.10$

$0.00-0.05$

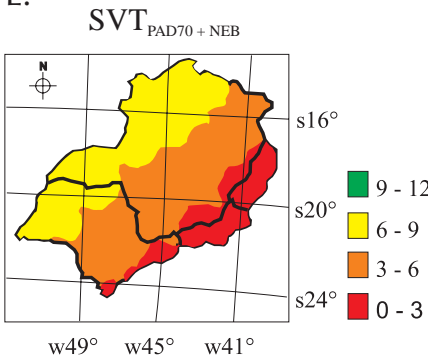

Esses resultados se devem às características das simulações que se baseiam em modelo de transferência de calor e massa em regime permanente, no qual a análise foi realizada para cada hora do período estudado (10 e 18 h). A adoção da análise em regime transiente poderá modificar sensivelmente os resultados das simulações, tornando-as mais realísticas. 
A.
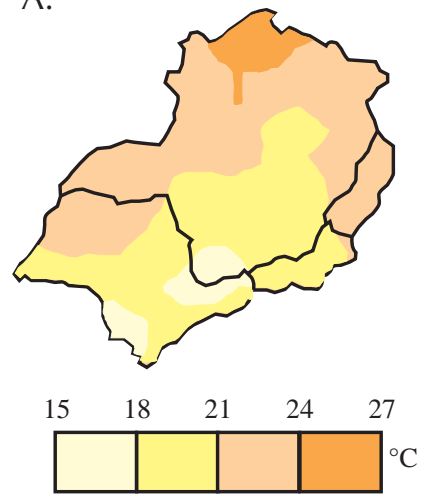

Fonte: INMET 1931/1990

Figura 3. Comportamentos espaciais da temperatura do ar média (tbs, $\left.{ }^{\circ} \mathrm{C}\right)$ (A) e da umidade relativa média (UR, \%) (B) para a região Sudeste do Brasil, no mês de setembro, adaptado de INMET (2006)

Apesar dos resultados da Tabela 1 representarem as médias encontradas para o ITU ao longo do ano, na região Sudeste, desempenhos melhores ou piores dos SRE podem ser observados ao se estudar o comportamento anual do ITU para locais específicos (Figuras 4 e 5). Enquanto para um local situado no norte de Minas (Figura 4A), se pode obter reduções de ITU de até 2,0, 3,3 e 3,4 para $\mathrm{SVT}_{\text {PAD40, }}$, $\mathrm{SVT}_{\mathrm{PAD70}}$ e $\mathrm{SVT}_{\mathrm{PAD70+NEB}}$, respectivamente, em outro local situado na mesorregião norte-fluminense (Figura 5A), considerada quente e úmida, as reduções são inferiores a 0,1 para as três configurações de SRE. Valores intermediários de redução de ITU são constatados para outros pontos localizados nas mesorregiões do Triângulo Mineiro/Alto Paranaíba, Metropolitana de São Paulo e Metropolitana de Belo Horizonte. Análise similar pode ser feita para CRD, GPD e CA, na qual diferenças mínimas resultantes da ação dos sistemas foram observadas para o local situado na mesorregião do norte-fluminense, enquanto diferenças mais evidentes foram notadas para o local situado na mesorregião do norte de Minas Gerais.

A análise do ambiente térmico pode ser ainda mais específica ao se analisar um galpão específico em determinada localidade (Figura 6). Pela Figura 6A, pode-se observar que os melhores ambientes térmicos são obtidos pelo uso do $\mathrm{SVT}_{\mathrm{PAD70+NEB}}, \mathrm{SVT}_{\mathrm{PAD70}}, \mathrm{SVT}_{\mathrm{NEB}}$ e SVT, nessa ordem. Neste estudo de caso se verifica que, enquanto no sistema $\mathrm{SVT}_{\mathrm{PAD70+NEB}} 59 \%$ do galpão estão em condições de conforto térmico (ITU < 74 e $50 £$ UR $£$ 70), nos demais, SVT PAD70, $_{\text {, }}$ $\mathrm{SVT}_{\mathrm{NEB}}$ e SVT as porcentagens foram de 53, 0 e $0 \%$, respectivamente (Figura 6).

\section{Desempenho produtivo das aves}

O comportamento mensal dos CRD, GPD e CA médios para SVT e seus respectivos aumentos (CRD e GPD) e redução (CA), em função das três diferentes configurações dos SRE simulados (SVT PAD40, $_{\text {SVT }}$ PAD70 e SVT PAD70+NEB $_{\text {), }}$ para a região Sudeste, é mostrado na Tabela 1, na qual se terá, com uso do SVT, que as aves apresentaram desempenho pouco variável, sendo melhor nos meses com temperaturas mais amenas (abril a julho); para o período de
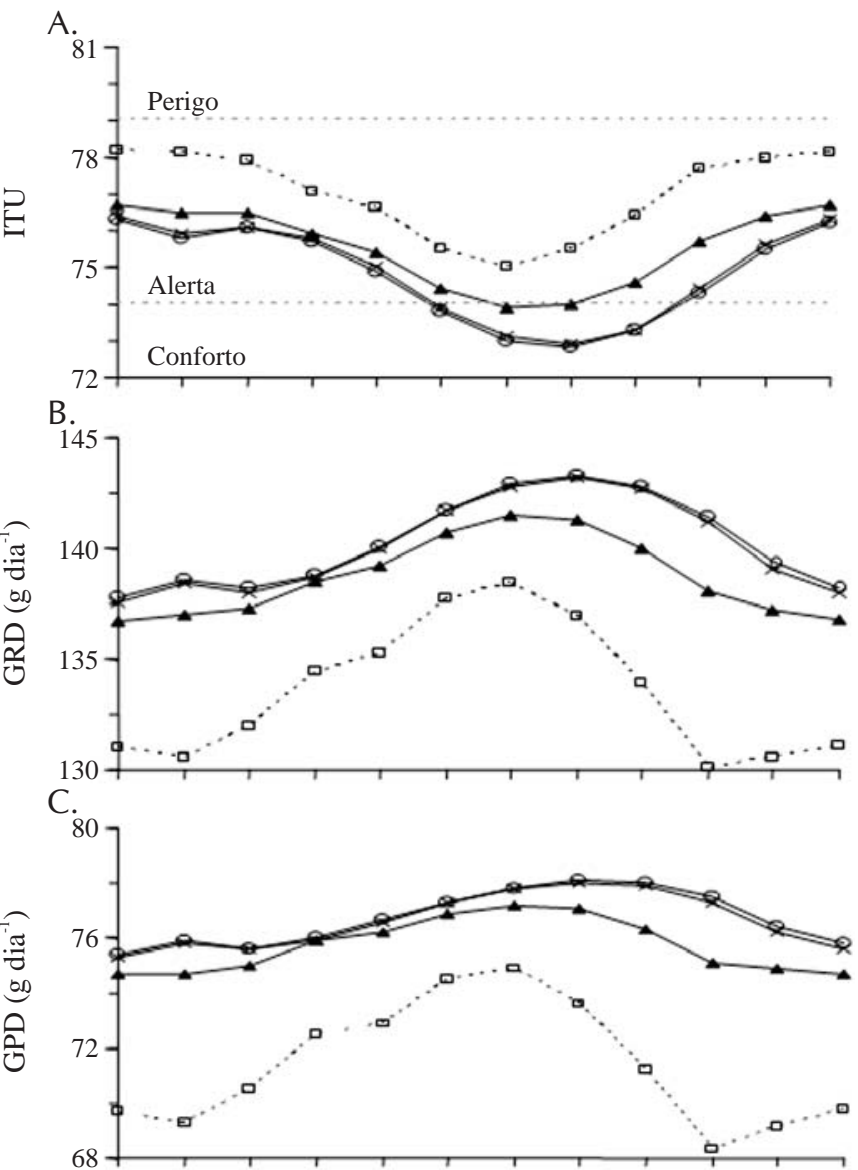

D.

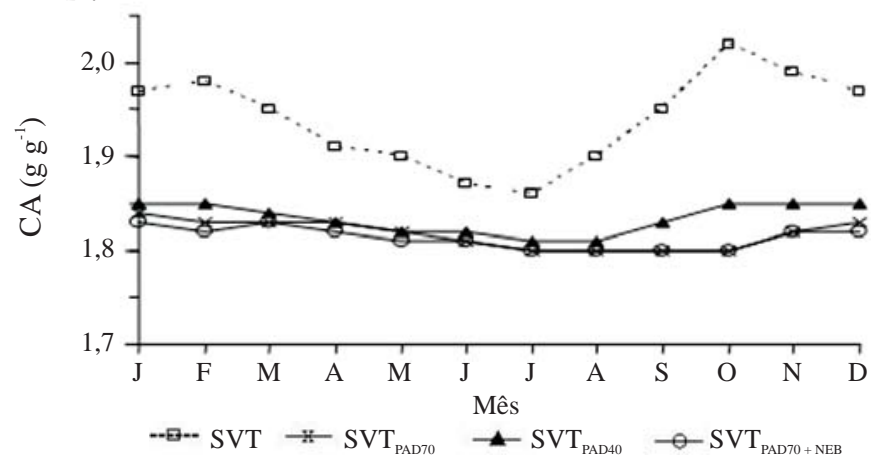

Figura 4. Valores médios de ITU, CRD, GPD e CA estimados para os tratamentos $\mathrm{SVT}, \mathrm{SVT}_{\text {PAD40, }}, \mathrm{SVT}_{\mathrm{PAD} 70}$ e $\mathrm{SVT}_{\mathrm{PAD} 70+\mathrm{NEB}}$ para um local situado na mesorregião do norte de Minas (Lat. $16^{\circ} 11^{\prime} 24^{\prime \prime} \mathrm{S}$ e Long. $\left.43^{\circ} 07^{\prime} 30^{\prime \prime} \mathrm{O}\right)$, para o período compreendido entre 1980 e 2001

verão, os valores médios simulados foram de 133,8 a $134,6 \mathrm{~g} \mathrm{~d}^{-1}$ para o CRD, 72,7 a 73,5 $\mathrm{g} \mathrm{d}^{-1}$ para GPD e 1,86 a $1,88 \mathrm{~g} \mathrm{~g}^{-1}$ para CA.

Ao se adotar os SRE, o ambiente térmico no interior do galpão tornou-se mais confortável e, assim, as aves responderam com aumento do CRD que, combinado com menor gasto de energia para dissipar calor, contribuiu para o aumento do GPD e redução da CA, ou seja, melhoraram seu desempenho. Os meses de setembro e outubro, foram novamente aqueles responsáveis pelos maiores benefícios do uso de SREs. Em setembro, as aves apresentaram aumento do CRD de 4,7 $\pm 3,6 \mathrm{~g} \mathrm{~d}^{-1}, 6,1 \pm 5,0 \mathrm{~g} \mathrm{~d}^{-1}$ e $6,1 \pm 5,0 \mathrm{~g} \mathrm{~d}^{-1}$, aumento do GPD de 4,0 $\pm 3,2 \mathrm{~g} \mathrm{~d}^{-1}, 4,9 \pm 4,2 \mathrm{~g} \mathrm{~d}^{-1}$ e $5,0 \pm$ 

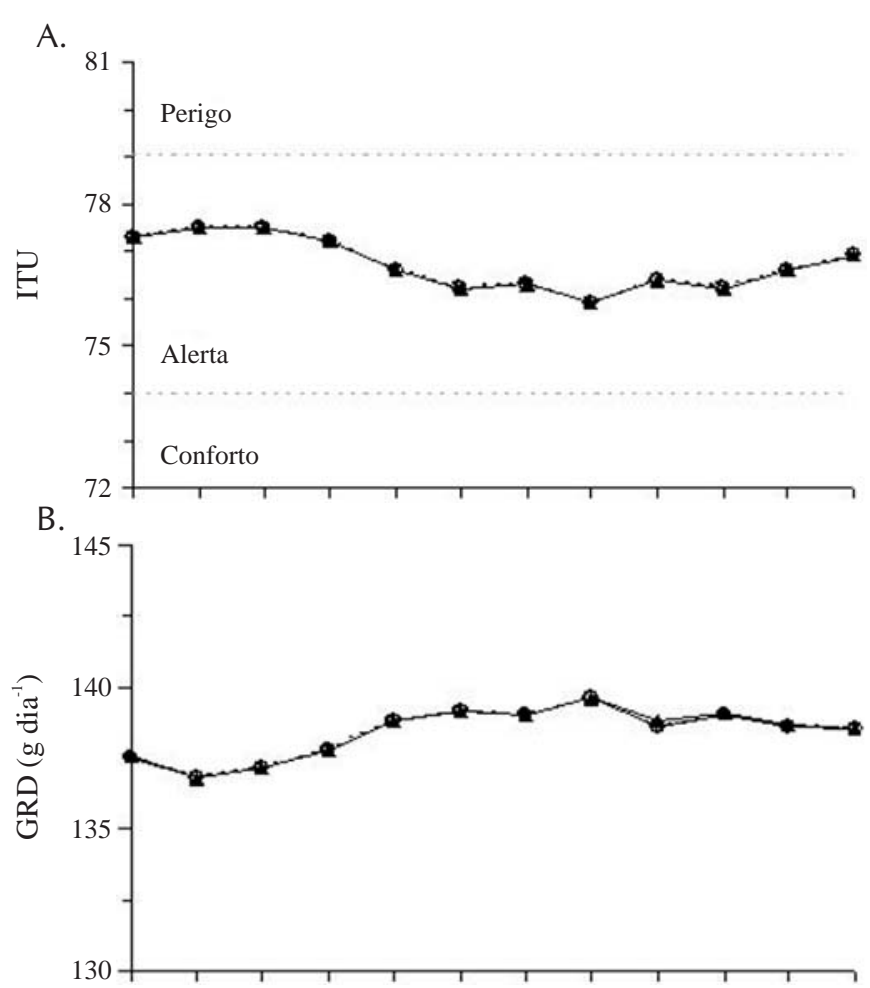

C.

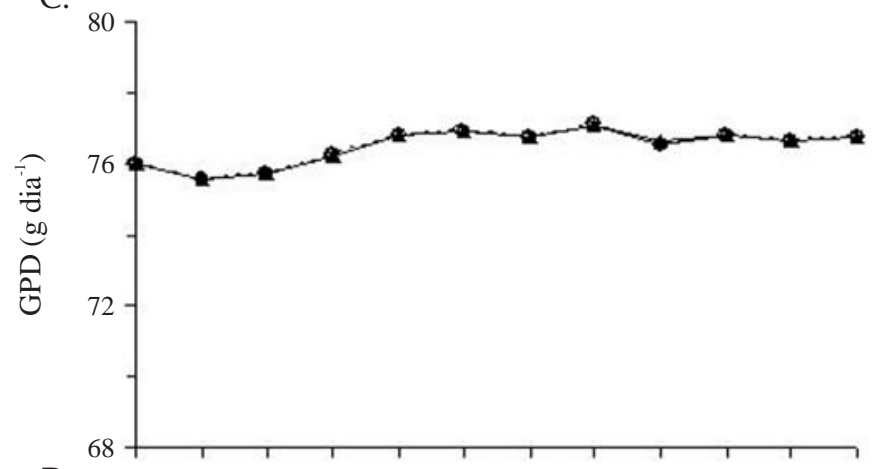

D.

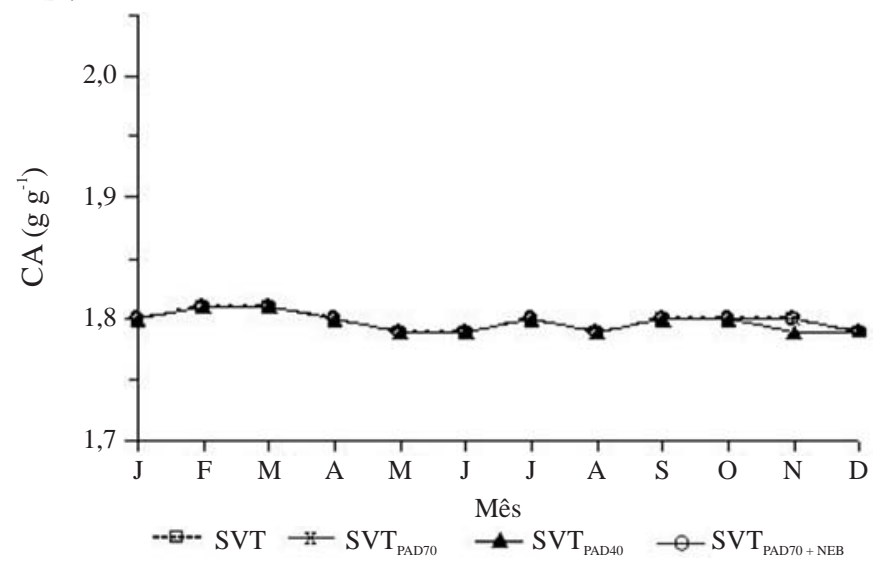

Figura 5. Valores médios de ITU, CRD, GPD e CA estimados para os tratamentos SVT, SVT $\mathrm{PAD}_{00}, \mathrm{SVT}_{\text {PAD70 }}$ e $\mathrm{SVT}_{\text {PAD70+NEB }}$ para um local situado na mesorregião do norte-fluminense (Lat. $21^{\circ} 54^{\prime} 16^{\prime \prime} \mathrm{S}$ e Long. $41^{\circ} 15^{\prime} 00^{\prime \prime}$ O), para o período compreendido entre 1980 e 2001

$4,2 \mathrm{~g} \mathrm{~d}^{-1}$ e redução da CA de $0,10 \pm 0,08 \mathrm{~g} \mathrm{~g}^{-1}, 0,12 \pm$ $0,10 \mathrm{~g} \mathrm{~g}^{-1}$ e $0,12 \pm 0,10 \mathrm{~g} \mathrm{~g}^{-1}$ para $\mathrm{SVT}_{\mathrm{PAD} 40}, \mathrm{SVT}_{\mathrm{PAD} 70}$ e $\mathrm{SVT}_{\mathrm{PAD} 70+\mathrm{NEB}}$, respectivamente.

Nos meses de março a junho se observou que, no ge-

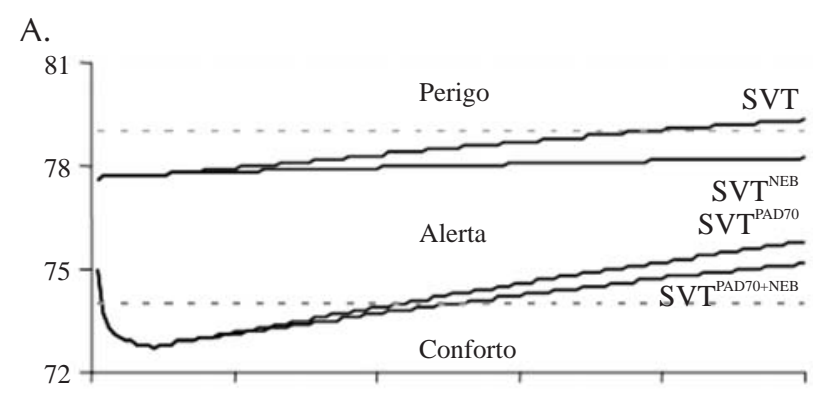

B.

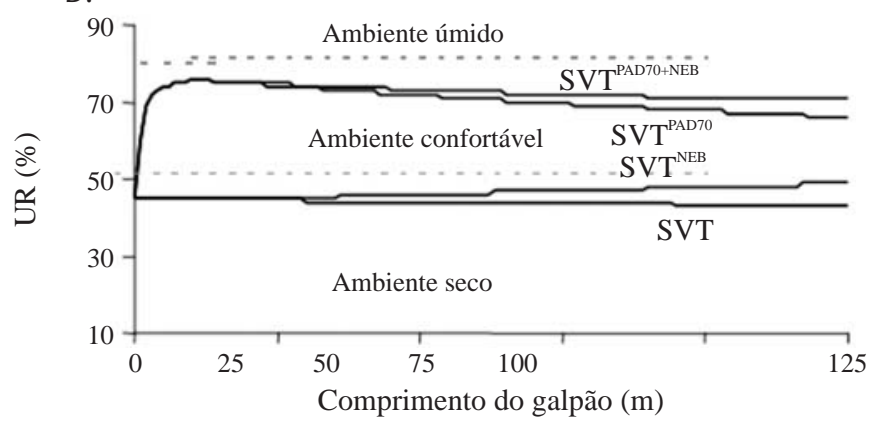

Figura 6. Comportamentos do ITU (A) e da UR (B), ao longo do comprimento do galpão, para os tratamentos SVT, $S T_{\text {PAD40, }}, S_{\text {PAD70 }}$ e $\mathrm{SVT}_{\text {PAD70+NEB }}$

ral, o desempenho médio das aves foi melhor para o tratamento $\mathrm{SVT}_{\mathrm{PAD} 40}$. Este comportamento é devido do desligamento mais freqüente do $\mathrm{SRE}$ nos tratamentos $\mathrm{SVT}_{\mathrm{PAD} 70}$ e $\mathrm{SVT}_{\mathrm{PAD} 70+\mathrm{NEB}}$ em relação ao $\mathrm{SVT}_{\mathrm{PAD} 40}$ pois, de acordo com o manejo adotado nesses sistemas e caso a UR alcance valores maiores ou iguais a $80 \%$, o SRE é desligado.

Nota-se, através da Figura 2, que o SRE foi mais bem aproveitado em partes das mesorregiões Norte e Noroeste de Minas, Triângulo Mineiro/Alto Paranaíba, São José do Rio Preto e Araçatuba, com aumentos de CRD e GPD e reduções de CA que chegaram a 9 a $12 \mathrm{~g} \mathrm{~d}^{-1}, 6$ a $9 \mathrm{~g} \mathrm{~d}^{-1}$ e 0,15 a $0,20 \mathrm{~g} \mathrm{~g}^{-1}$, respectivamente.

Para a maioria das mesorregiões dos estados do Rio de Janeiro e Espírito Santo, mesorregião Metropolitana de São Paulo e parte das mesorregiões divisoras de Minas Gerais com os estados do Rio de Janeiro e Espírito Santo, pouco beneficio trouxe o uso dos SREs, encontraram-se reduções de CA de 0 a $0,05 \mathrm{~g} \mathrm{~g}^{-1}$ e aumentos de CRD e GPD de 0 a $3 \mathrm{~g} \mathrm{~d}^{-1}$. Similarmente ao ITU, os efeitos benéficos do uso dos SRE, em relação ao desempenho das aves, aumentam na medida em que se deslocam do litoral para continente o, no sentido noroeste.

Na Figura 4 os maiores aumentos do CRD e GPD e reduções de CA, proporcionados pelo uso dos SREs em relação ao SVT, foram de $11,3 \mathrm{~g} \mathrm{~d}^{-1}, 9,2 \mathrm{~g} \mathrm{~d}^{-1}$ e $0,22 \mathrm{~g} \mathrm{~g}^{-1}$, respectivamente, para um local específico na mesorregião Norte de Minas, indicando que a referida região possui os melhores potenciais de aumento do desempenho das aves, comparado com as demais mesorregiões dos estados do Sudeste; por outro lado, o uso de SREs em galpões localizados na mesorregião Norte-Fluminense auxilia pouco no desempenho das aves (Figura 5). 


\section{CONCLUSÕES}

1. Altos valores de temperatura do ar associados aos das altas umidades relativas que ocorrem nos estados do Espírito Santo, Rio de Janeiro e parte do litoral paulista, são limitantes ao uso de sistemas de resfriamento evaporativo.

2. Os meses de setembro e outubro foram aqueles com maiores potenciais de redução de ITU, refletindo beneficamente sobre o desempenho das aves, exceto para a maioria das mesorregiões dos estados do Espírito Santo e Rio de Janeiro, parte do Litoral Sul-Paulista, Zona da Mata e Vale do Rio Doce.

3. As mesorregiões mais beneficiadas com o uso de resfriamento evaporativo são parte do Norte e Noroeste de Minas, do Triângulo Mineiro/Alto Paranaíba, São José do Rio Preto e Araçatuba.

4. Durante os meses mais quentes e menos úmidos, o sistema de resfriamento evaporativo com eficiência de $70 \%$ $\left(\mathrm{SVT}_{\mathrm{PAD70}}\right)$ apresentou melhor desempenho que aquele com $40 \%\left(\mathrm{SVT}_{\mathrm{PAD} 40}\right)$.

5. O uso do sistema $\mathrm{SVT}_{\mathrm{PAD} 70+\mathrm{NEB}}$ é recomendo para a região Sudeste do Brasil, em razão de proporcionar melhores condições de conforto às aves, uma vez que somente o uso da ventilação não foi suficiente para lhes garantir conforto térmico às aves no período estudado, para as horas mais quentes do dia.

\section{AGRADECIMENTOS}

Os autores expressam seus agradecimentos à FAPEMIG, à CAPES e ao CNPq, pelo financiamento do projeto e pela concessão de uma bolsa de mestrado e PIBIC, respectivamente.

\section{LITERATURA CITADA}

Baêta, F. C.; Souza, C. F. Ambiência em edificações rurais: conforto térmico animal. Viçosa: UFV, 1997. 246p

Baião, N. C. Efeitos da alta densidade populacional sobre o ambiente das instalações avícolas. In: Simpósio Internacional sobre Ambiência e Instalações na Avicultura Industrial, 1995, São Paulo. Anais... Campinas: FACTA, 1995. p.67-75.

Bueno, L. G. F. Avaliação da eficiência energética e do conforto térmico em instalações de frango de corte. Campinas: UNICAMP, 2004. 100p. Dissertação Mestrado

Câmara, G.; Souza, R. C. M.; Freitas, U. M.; Garrido, J. "Spring: Integrating remote sensing and GIS by object-oriented data modelling”. Computers \& Graphics, v.20, n.3, p.395-403, 1996.

Carvalho, V. F. Modelagem e zoneamento do ambiente térmico para avaliar o desempenho de frangos de corte criados em galpões climatizados. Lavras: UFLA, 2006. 77p. Dissertação Mestrado

Corria, M. E.; Nääs, I. A. Adapting tunnel ventilation for high density broiler production - a case study. In: International Livestock Environment Symposium, 6, 2001, Louisville. Proceedings... Louisville: ASAE, 2001. p.461-467.

Curtis, S. E. Environmental management in animal agriculture. The Iowa State University: Ames, 1983. 410p.
Gates, R. S.; Zhang, H.; Colliver, D. G.; Overhults, D. G. Regional variation in temperature humidity index for poultry housing. Transactions of the ASAE, v.38, n. 1, p.197-205, 1995.

IBGE - Instituto Brasileiro de Geografia e Estatística. Mesorregiões. http://www.ibge.gov.br. 20 Jul. 2006.

INMET - Instituto Nacional de Meteorologia Normais climatológicas. http://www.inmet.gov.br. 17 Jul. 2006.

INPE - Instituto Nacional de Pesquisas Espaciais. Sistema de processamento de informações georeferenciadas - SPRING. http:/ /www.dpi.inpe.br/spring. 17 Jul. 2006.

Jácome, I. M. T. D.; Furtado, D. A.; Leal, A. F.; Silva, J. H. V.; Moura, J. F. P. Avaliação de índices de conforto térmico de instalações para poedeiras no nordeste do Brasil. Revista Brasileira de Engenharia Agrícola e Ambiental, v.11, n.5, p.537-531, 2007.

Medeiros, C. M. Ajuste de modelos e determinação de índice térmico ambiental de produtividade para frangos de corte. Viçosa: UFV, 2001. 115 p. Tese Doutorado

Medeiros, C. M.; Baêta, F. C.; Oliveira, R. F. M.; Tinôco, I. F. F. T.; Albino, L. F. T.; Cecon, P. R. Efeitos da temperatura, umidade relativa e velocidade do ar em frangos de corte. Engenharia na Agricultura, v.13, n.4, 277-286, 2005.

Oliveira, L. M. F. Efeitos climáticos no conforto térmico animal e humano. Lavras: UFLA, 2004. 104p. Dissertação Mestrado

Oliveira, L. M. .;Yanagi Júnior, T.; Ferreira, E.; Carvalho, L. G.; Silva, M. P. Zoneamento bioclimático da região Sudeste do Brasil para o conforto térmico animal e humano. Engenharia Agrícola, v.26, p.823-831, 2006.

Sarmento, L. G. V.; Dantas, R.; Furtado, D.; Nascimento, J. W. B.; Silva, J. H. V. Efeito da pintura externa do telhado sobre o ambiente climático e o desempenho de frangos de corte. Agropecuária Técnica, v.26, n.2, p.117-112, 2005.

Sartor, V.; Baêta, F. C.; Luz, M. L; Orlando, R. C. Sistemas de resfriamento evaporativo e o desempenho de frangos de corte. Scientia Agricola, v.58, n.1, p.17-20, 2001.

Severo, J. C. A.; Yanagi Junior, T.; Santos, S. N. M.; Silva, J. P.; Borges, G.; Teixeira, V. H. Desenvolvimento de um software para predição do ambiente térmico no interior de galpões agropecuários. Revista Brasileira de Agroinformática, v.5, n.2, p.59-72, 2003.

Silva, E. T.; Leite, D. G.; Yuri, F. M.; Nery, F. S.; Rego, J. C. C.; Zanatta, R. A.; Santos, S. A.; Moura, V. V. Determinação do índice de temperatura e umidade (ITU) para produção de aves na mesorregião metropolitana de Curitiba - PR. Revista Acadêmica: Ciências Agrárias e Ambientais, v.2, n.3, p.47-60, 2004.

Silva, M. P.; Baêta, F. C; Tinôco, I. F. F.; Zolnier, S.; Ribeiro, A. Evaluation of the evaporative cooling usage potential for the Southeast region of Brazil with a simplified model for the estimation of the energy balance in sheds for broiler chickens. In: International livestock environment symposium, 7., 2005, Beijing. Proceedings... Beijing: ASABE, 2005. p.143-150.

Stowell, R. R.; Gooch, C. A.; Inglis, S. Performance of tunnel ventilation for freestall dairy facilities as compared to natural ventilation with supplemental cooling fans. In: International livestock environment symposium, 6, 2001, Louisville. Proceedings... Louisville: ASAE, 2001. p.29-40.

Thom, E. C. The discomfort index. Weatherwise, v.12, n.1, p.57-60, 1959.

R. Bras. Eng. Agríc. Ambiental, v.13, n.3, p.358-366, 2009. 
Tinôco, I. F. F. Efeito de diferentes sistemas de acondicionamento de ambiente e níveis de energia metabolizável na dieta, sobre o desempenho de matrizes de frangos de corte, em condições de verão e outono. Belo Horizonte: UFMG, 1996. 169p. Tese Doutorado.

Tinôco, I. F. F; Figueiredo, J. L. A.; Santos, R. C.; Silva, J. N.; Pugliesi, N. L. Placas porosas utilizadas em sistemas de resfriamento evaporativo. Engenharia na Agricultura, v.12, n.1, p.17-23, 2004.

Turco, S. H. N.; Silva, T. G. F; Santos, L. F. C; Ribeiro, P. H. B.; Araújo, G. G. L.; Holanda Jr., E. V.; Aguiar, M. A. Zoneamento bioclimático para vacas leiteiras no estado da Bahia. Engenharia Agrícola, v.26, n.1, p.20-27, 2006.
Yanagi Júnior, T.; Xin, H.; Gates, R. S. Optimization of partial surface wetting to cool caged laying hens. Transactions of the ASAE, v.45, n.4, p.1091-1100, 2002.

Zolnier, S. Avaliação de modelos para estimativa dos valores médios horários do índice de temperatura e umidade. Revista Engenharia na Agricultura, v.5, n.16, p.1-17, 1996.

Zulovich, J. M.; Deshazer, J. A. Estimative egg production declines at high environmental temperatures and humidities. St. Joseph: ASAE, 1990. Paper n.904021. 